\title{
Synthesis of Boron Nanorods by Smelting Non-Toxic Boron Oxide in Liquid Lithium
}

\author{
Amartya Chakrabarti, ${ }^{1,2}$ Tao Xu, ${ }^{1}$ Laura K. Paulson, ${ }^{1}$ Kate J. Krise, ${ }^{1}$ John A. Maguire, ${ }^{2}$ \\ and Narayan S. Hosmane ${ }^{1}$ \\ ${ }^{1}$ Department of Chemistry and Biochemistry, Northern Illinois University, DeKalb, IL 60115, USA \\ ${ }^{2}$ Department of Chemistry, Southern Methodist University, Dallas, TX 75275, USA \\ Correspondence should be addressed to Tao Xu, txu_mail@yahoo.com
}

Received 25 November 2009; Accepted 16 February 2010

Academic Editor: Zhi-Li Xiao

Copyright (C) 2010 Amartya Chakrabarti et al. This is an open access article distributed under the Creative Commons Attribution License, which permits unrestricted use, distribution, and reproduction in any medium, provided the original work is properly cited.

In contrast to the conventional bottom-up syntheses of boron nanostructures, a unique top-down and greener synthetic strategy is presented for boron nanorods involving nontoxic boron oxide powders ultrasonically smelted in liquid lithium under milder conditions. The product was thoroughly characterized by energy dispersive X-ray analysis, atomic emission spectroscopy, thermogravimetric analysis and, UV-Vis spectroscopy, including structural characterization by transmission electron microscopy (TEM).

\section{Introduction}

Elemental boron, by virtue of its unique electron-deficient nature, superhardness (Knoop: 2160-2900), and high melting point $\left(\sim 2200^{\circ} \mathrm{C}\right)$, has been found to be very useful in a diverse range of industrial applications including semiconductors, wear-resistant additives and protective coatings, as well as high-temperature devices [1]. Boron-based nanomaterials have attracted a wide range of interests in a number of applications [2]. For example, boron-doped diamond nanopowder has been synthesized and its electrochemical behavior studied; it is proved to be an excellent electrocatalyst support material [3] and also an electrode material [4]. Boron-based nanoparticles are now being extensively investigated as a boron source in boron neutron capture therapy [58]. The high surface-to-volume ratio of boron nanoparticles coupled with boron's high energy content makes such nanoparticles attractive as fuel additives in conventional hydrocarbon fuels for airbreathing propulsion systems $[9$, 10]. Their robust thermal and electrical stability, compared to metal nanowires, should make 1-dimentional boron nanostructures such as nanowires, nanorods, nanobelts, or nanotubes, as promising materials for nanoscale electrically conducting interconnects in nanoelectronic devices $[11,12]$.
Despite the rapid growth of their applications, limited progress has been made in the synthesis of boron nanomaterials. Nearly all synthetic methods for boron nanomaterials are based on the bottom-up strategy that inevitably involves the usage of gas phase decomposition of the highly toxic and flammable precursory gases under drastic reaction conditions. For example, synthesis of amorphous boron nanoparticles from furnace heating of diborane at $700^{\circ} \mathrm{C}$ was reported in early 1950s [13]. The product had a large size distribution. Later, laser heating and arc discharge was employed to narrow down the size distribution of the boron nanoparticles $[14,15]$. On the other hand, synthesis of boron nanowires was first reported by Ruoff and coworkers through the decomposition of diborane on $\mathrm{NiB}$ powder as a catalyst at $1100^{\circ} \mathrm{C}$ [16]. In a similar fashion, boron nanorods were fabricated by reduction of diborane on a thin film of $\mathrm{Al}$ catalyst over silicon substrates at the temperature range of $750-900^{\circ} \mathrm{C}$ [17]. While boron nanoribbons were prepared recently through catalyst-free pyrolysis of diborane at 630 $750^{\circ} \mathrm{C}$ [18], crystalline boron nanobelts were formed via laser ablation techniques at temperatures from 700 to $1000^{\circ} \mathrm{C}$ [19]. Alternatively, $\mathrm{BCl}_{3}$ was used as a boron precursor to synthesize boron nanotubes at $870^{\circ} \mathrm{C}$ with hydrogen gas as reducing agent [20]. To date, some progress has been made 


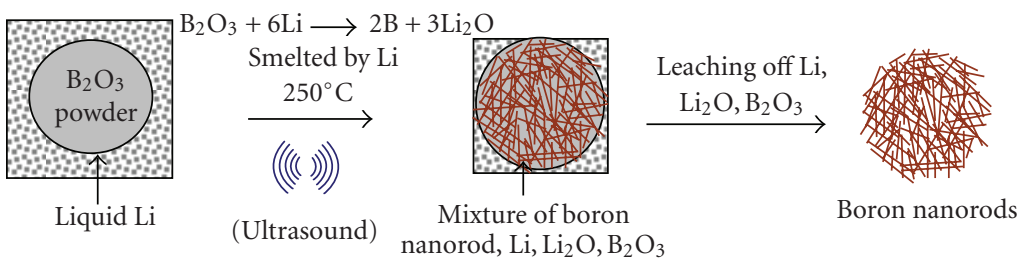

FIGURE 1: Schematic representation of the formation of boron nanorods by ultrasound-assisted smelting $\mathrm{B}_{2} \mathrm{O}_{3}$ powders in liquid lithium.

in moderating the reaction conditions including a room temperature solution-based synthesis of boron nanoparticles through reduction of boron tribromide utilizing sodium naphthalenide [21]. Nonetheless, the boron precursors in all of the reported syntheses are highly toxic. Therefore, a greener and energy-efficient synthetic strategy is warranted for the synthesis of boron nanostructures.

In this context, it is important to note that a top-down method was reported for nanoporous platinum from its oxide [22]. In this method, lithium was used to intercalate through the matrix of $\mathrm{PtO}_{2}$ microparticles electrochemically via the reductive smelting process: $\mathrm{PtO}_{2}+4 \mathrm{Li} \rightarrow \mathrm{Pt}+$ $2 \mathrm{Li}_{2} \mathrm{O}$. The by-product $\mathrm{Li}_{2} \mathrm{O}$ could be removed by extraction in acidic aqueous solution or water in order to isolate nanoporous platinum. We also demonstrated a bottom-up strategy for the synthesis of $\mathrm{Pt}$ nanoparticles directly from bulk $\mathrm{Pt}$ using $\mathrm{Li}$ as a solvent [23]. These results posed a question whether this methodology could be extended to produce boron nanostructures as well. Although the magnesium metal has been known to be a good reducing agent in the production of bulk boron from boron oxide [24], in the production of boron nanostructures we chose to utilize the lithium metal due to the fact that $\mathrm{Li}$ (reduction potential $=-3.07 \mathrm{~V}$ ) is a much stronger reducing agent than $\mathrm{Mg}$ (reduction potential $=-2.37 \mathrm{~V}$ ) and assures the feasibility of the reaction. In addition, the corrosive intercalation of liquid $\mathrm{Li}$ into other materials is a notorious flaw when it is used as a coolant in nuclear reactors $[25,26]$, but is taken here as an advantage to produce nanostructured boron from boron oxide, which is a nontoxic starting material that is conveniently available from the major natural sources of boron, for example, borax. Figure 1 illustrates the synthetic strategy of the top-down methodology.

\section{Experimental Section}

2.1. Synthesis of Boron Nanorods. $500 \mathrm{mg}$ of $\mathrm{B}_{2} \mathrm{O}_{3}$, previously dried overnight at $110^{\circ} \mathrm{C}$ under vacuum, was thoroughly ground inside an argon glove-box using a mortar and pestle. Subsequently, metallic lithium was weighed in a 6fold molar ratio to $\mathrm{B}_{2} \mathrm{O}_{3}$, in a nickel crucible and melted at $200^{\circ} \mathrm{C}$. In the $\mathrm{Ar}$ glovebox, $\mathrm{B}_{2} \mathrm{O}_{3}$ powders were added under sonication into the molten $\mathrm{Li}$ and temperature of the mixture was increased up to $250^{\circ} \mathrm{C}$ and maintained at that temperature under a tip-sonication for 2 hours. The mixture was cooled down to room temperature and taken out of the glovebox. Methanol was added to this mixture in order to remove any unreacted lithium. The resulting solution was filtered, washed thoroughly with hot water, dilute potassium hydroxide solution, dilute hydrochloric acid solution followed by repeated washing with cold water. The brownish product was then dried under vacuum at $70^{\circ} \mathrm{C}$ for 24 hours before characterization. Approximately $150 \mathrm{mg}$ of product was collected after complete drying in vacuo.

The solid product was subjected to all available characterization techniques. Transmission electron microscopy (TEM) imaging of the purified product was done by a Hitachi H-600 $(75 \mathrm{kV})$ instrument and elemental analysis of the samples was done through energy dispersive X-ray (EDX) studies using a field emission scanning electron microscope (Hitachi S-4700-II), and identified the product to be the boron nanorods.

Since the EDX spectrum has not been able to determine any lithium traces present in the boron nanorods sample, we ran an atmoic emission studies using the atomic absorption spectrometer (AAS, Perkin Elmer 3110). About $20 \mathrm{mg}$ of boron nanorods sample was dispersed in $50 \mathrm{~mL}$ of deionized water. The atomic emmision was measured for the sample at the characteristic emission wavelength of lithium of $670.8 \mathrm{~nm}$. To get the lithium concentration in the sample, a calibration plot was generated by running several samples of lithium solutions with known concentrations. Four different solutions of known lithium nitrate ( $1 \mathrm{ppm}, 5 \mathrm{ppm}, 10 \mathrm{ppm}$, and $15 \mathrm{pmm}$ ) were prepared by diluting the stock customgrade standard lithium solution $(995 \pm 2 \mu \mathrm{g} / \mathrm{mL})$ from Inorganic Ventures Inc. in deionized water.

Thermogravimetric analysis (TGA, Perkin-Elmer Pyris 1) was performed on the boron nanorods samples. A known amount of sample (perivously vacuum dried at $70^{\circ} \mathrm{C}$ for 48 hours) was subjected to a temperature scan from $25^{\circ} \mathrm{C}$ to $700^{\circ} \mathrm{C}$ and the corresponding weight losses were recorded.

UV-Vis scpectroscopy (Perkin Elmer Lambda 19) was also carried out on the boron nanorod sample. Boron nanorod sample solution was prepared as a deionized water dispersion using a quartz cell. Deionized water was used as blank reference in the experiment. The solution was subjected to a wavelength scan from $700 \mathrm{~nm}$ to $190 \mathrm{~nm}$.

\section{Results and Discussion}

In the preparatory process of the boron nanorod, the purification part was the key to the synthetic success. Apparently, it was crucial to avoid any formation of lithium borides in the synthesis or at least destroy such a contaminant in the product with proper treatment and washing sequences. According to some earlier work by Serebryakova et al. 


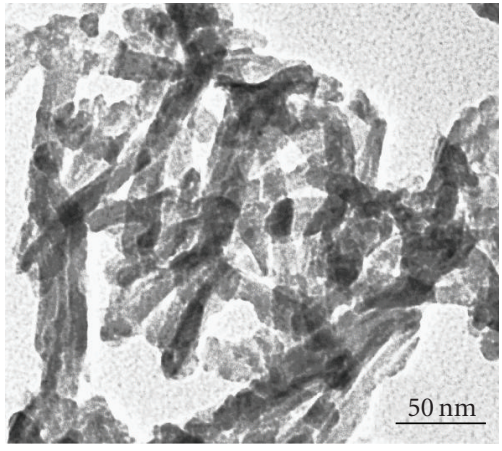

(a)

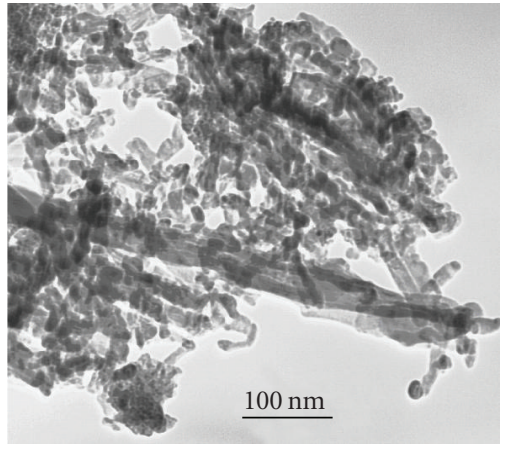

(b)

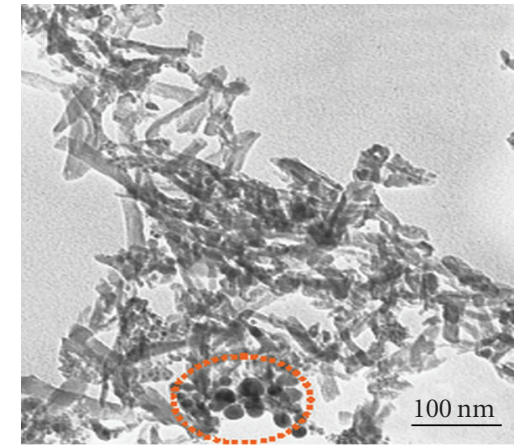

(c)

FIGURE 2: TEM images of boron nanorods showing abundant nanorod formation of varying length and diameters (a) bundles of nanorods; (b) some long nanorods; (c) nanorods of shorter lengths and the spherical boron nanoparticles (highlighted in the circular part).

lithium diboride is not very stable and is easily soluble in methanol; it also decomposes in weak mineral acid solutions [27]. On the other hand, lithium tetraboride is not stable in potassium hydroxide solution. Thus, these observations guided the purification protocol applied in the synthesis of boron nanorods described above. To avoid the vigorous reaction of water with lithium, methanol was first added after completion of the reaction. One of the reasons for not washing the product immediately with water is to avoid the formation of lithium hydroxide which is capable of etching the nickel crucible. The $\mathrm{B}_{2} \mathrm{O}_{3}$, being soluble in hot water, was easily removed by washing with hot water. While potassium hydroxide solution was used to remove any lithium tetraboride, dilute $\mathrm{HCl}$ solution was used to take away the lithium diborides, if any, as well as to neutralize the basic condition of the solution. Washings were repeated until the product was free of any residual acid.

Figure 2 is the TEM images of the resulting boron materials. Apparently, the dominant feature is the rodlike structures (Figure 2(a)). The preferential formation of boron nanorods over nanoparticles must be associated with the streaming-like ultrasonic propagation [28, 29], which can transfer acoustic momentum efficiently to the liquid lithium [30], leading to a sort of directed lithiation in a $\mathrm{B}_{2} \mathrm{O}_{3}$ powder. While in a random insertion of lithum, the dominant resulting structure of boron is believed to be nanoparticles. The TEM images also suggest that the diameters of most of the nanorods exhibit a fairly narrow distribution, ranging between 20 and $40 \mathrm{~nm}$. The length of the rods varies and most of the length of the nanorods fall in the region between 80 and $200 \mathrm{~nm}$, but there are some longer nanorods of more than $500 \mathrm{~nm}$ (Figure 2(b)). Some spherical boron nanoparticles embedded in the boron nanorods (Figure 2(c)) were also observed. Nonetheless, we were able to produce boron nanorods in good yields (85\%) from $\mathrm{B}_{2} \mathrm{O}_{3}$ by this ultrasonic smelting method. This simple and greener process neither involves any harmful and toxic boron precursors nor does it require any severe reaction conditions.

Figure 3 shows the EDX spectrum of the specimen which clearly bears no evidence of containing any elements other

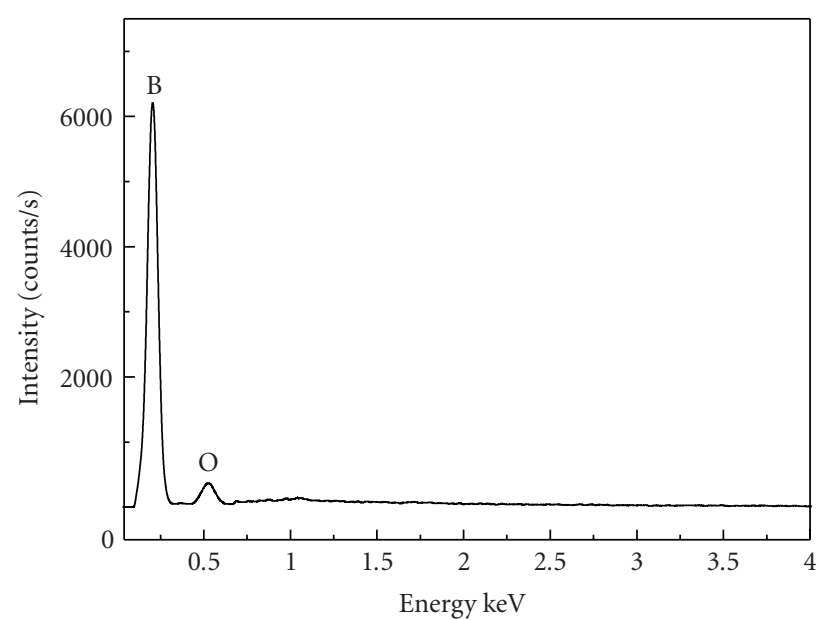

FIGURE 3: EDX spectrum of boron nanorod sample.

than boron (95 at.\%) and oxygen (5 at.\%). The very trace amount of oxygen found is due to the presence of the inevitable water moisture and oxygen absorbed on the boron nanorods or perhaps trace amount of unreacted $\mathrm{B}_{2} \mathrm{O}_{3}$.

The concentration versus signal intensity plot is depicted in Figure 4. When the signal intensity for the boron nanorods sample was fed into the calibration, bearing the trendline of $y=0.12 x$, the lithium concentration for the sample came out to be $1.83 \mathrm{ppm}$. The atomic ratio of lithium to boron in the sample thus calculated to be less than $0.95 \%$.

Figure 5 exhibits the TGA thermogram of boron nanorod sample. Under inert atmosphere (argon), the boron nanorods sample was thermally stable and did not show any significant weight loss within the experimental temperature range, which demonstrates the absence of any other vulnerable impurities in the prepared sample. However, when heated in air, the sample started to gain weight at temperature above $550^{\circ} \mathrm{C}$ due to the inevitable oxidation.

There are several reports on the theoretical calculation for the crystal structure and optical properties of boron crystals [31, 32]. The UV-Vis spectra of boron nanorod 


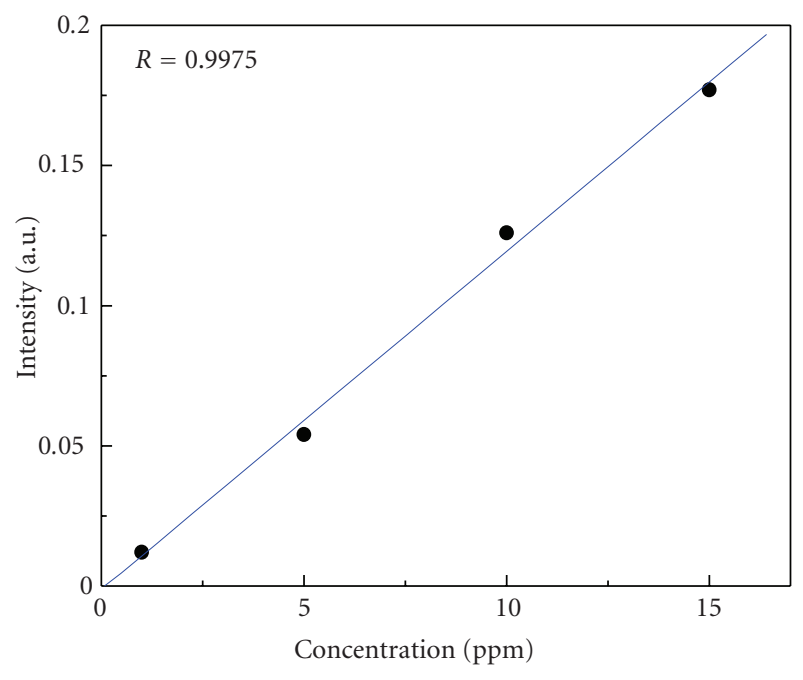

FIGURE 4: Calibration plot of emission versus Li concentration for atomic emission spectroscopy.

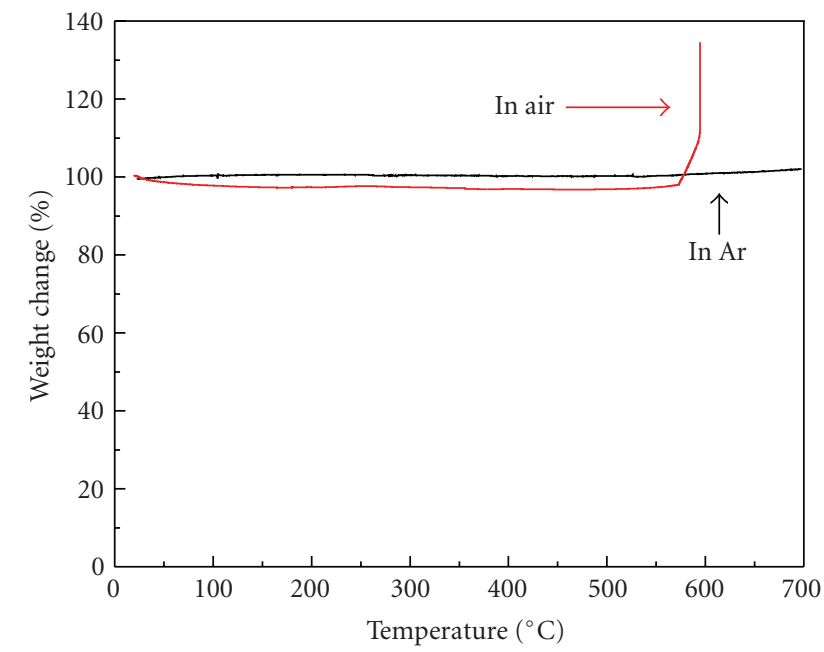

FIGURE 5: TGA thermogram of boron nanorods in Ar (black) and in air (red). The initial weight loss in air is due to the presence of moisture in the sample.

sample is demonstrated in Figure 6. The peak observed at $198 \mathrm{~nm}(6.26 \mathrm{eV})$ closely matches with the values reported by Yang et al. [17]. The peak threshold was observed around $225 \mathrm{~nm}$ (corresponding to $5.5 \mathrm{eV}$ ) which obtained its maximum at $198 \mathrm{~nm}(6.26 \mathrm{eV})$. Since quartz is transparent in the UV region, the peak is believed to be due to the boron sample.

\section{Conclusion}

We report here the first top-down synthesis of boron nanorods at low temperature and without usage of any catalysts or flammable and toxic gaseous precursors. Nontoxic boron oxide was reduced by lithum in this ultrasonication assisted synthesis. The purity of the product was confirmed by EDX spectroscopy, atomic absorption

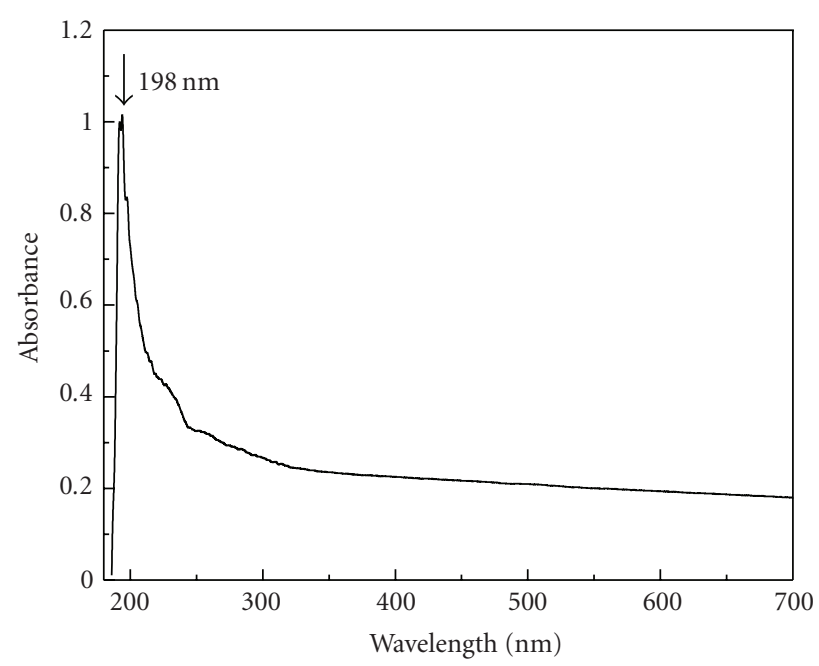

FIGURE 6: UV-Vis spectrum of boron nanorod sample.

spectroscopy, and thermogravimteric analysis; whereas the structural confirmation has been derived from transmission electron microscopy. Bundles of nanorods of a narrow range of diameters, along with some spherical particles, were observed under the microscope. Thus, this work demonstrates an alternative route for low temperature, nonhazardous large-scale synthesis of boron nanomaterials.

\section{Acknowledgments}

T.Xu acknowledges the support of the American Chemical Society Petroleum Research Fund (Type G 46374-G10). The sixth of author thanks the support of the National Science Fundation (CHE-0601023 and CHE-0906179) and the fifth of author thanks the Robert A. Welch Foundation (N-1322). The authors thank Ms. Lori Bross for help with the TEM. The sixth author gratefully acknowledges the second-time Humboldt Research Prize for US Senior Scientists from the Alexander von Humboldt Stiftung and the Inaugural Board of Trustees Professorship Award from Northern Illinois University.

\section{References}

[1] R. M Adams, Ed., Boron, Metallo-Boron, Compounds and Boranes, Interscience, New York, NY, USA, 1964.

[2] I. Boustani, A. Quandt, E. Hernández, and A. Rubio, "New boron based nanostructured materials," Journal of Chemical Physics, vol. 110, no. 6, pp. 3176-3185, 1999.

[3] A. E. Fischer and G. M. Swain, "Preparation and characterization of boron-doped diamond powder," Journal of the Electrochemical Society, vol. 152, no. 9, pp. B369-B375, 2005.

[4] J. B. Zang, Y. H. Wang, H. Huang, and W. Tang, "Electrochemical behavior of high-pressure synthetic boron doped diamond powder electrodes," Electrochimica Acta, vol. 52, no. 13, pp. 4398-4402, 2007.

[5] M. W. Mortensen, O. Björkdahl, P. G. Sørensen, et al., "Functionalization and cellular uptake of boron carbide naraoparticles. The first step toward $\mathrm{T}$ cell-guided boron 
neutron capture therapy," Bioconjugate Chemistry, vol. 17, no. 2, pp. 284-290, 2006.

[6] M. W. Mortensen, P. G. Sørensen, O. Björkdahl, M. R. Jensen, H. J. G. Gundersen, and T. Bjørnholm, "Preparation and characterization of Boron carbide nanoparticles for use as a novel agent in T cell-guided boron neutron capture therapy," Applied Radiation and Isotopes, vol. 64, no. 3, pp. 315-324, 2006.

[7] Z. Yinghuai, K. Cheng Yan, J. A. Maguire, and N. S. Hosmane, "Recent developments in boron neutron capture therapy (BNCT) driven by nanotechnology," Current Chemical Biology, vol. 1, no. 2, pp. 141-149, 2007.

[8] G. Ciofani, V. Raffa, A. Menciassi, and A. Cuschieri, "Folate functionalized boron nitride nanotubes and their selective uptake by glioblastoma multiforme cells: implications for their use as boron carriers in clinical boron neutron capture therapy," Nanoscale Research Letters, vol. 4, no. 2, pp. 113-121, 2009.

[9] K. Kleiner, "Powdered metal: the fuel of the future," New Scentist, no. 2522, pp. 34-37, 2005.

[10] G. Young, K. Sullivan, M. R. Zachariah, and K. Yu, "Combustion characteristics of boron nanoparticles," Combustion and Flame, vol. 156, no. 2, pp. 322-333, 2009.

[11] J. Kunstmann and A. Quandt, "Constricted boron nanotubes," Chemical Physics Letters, vol. 402, pp. 21-26, 2005.

[12] A. Gindulyte, W. N. Lipscomb, and L. Massa, "Proposed boron nanotubes," Inorganic Chemistry, vol. 37, no. 25, pp. 65446545, 1998.

[13] H. L. Johnston, H. N. Hersh, and E. C. Kerr, "Low temperature heat hapacities of inorganic solids. V. The heat capacity of pure elementary boron in both amorphous and crystalline conditions between 13 and $305^{\circ} \mathrm{K}$. Some free energies of formation," Journal of the American chemical society, vol. 73, no. 3, pp. 1112-1117, 1951.

[14] J. D. Casey and J. S. Haggerty, "Laser-induced vapour-phase syntheses of boron and titanium diboride powders," Journal of Materials Science, vol. 22, no. 2, pp. 737-744, 1987.

[15] P. Z. Si, M. Zhang, C. Y. You, et al., "Amorphous boron nanoparticles and $\mathrm{BN}$ encapsulating boron nano-peanuts prepared by arc-decomposing diborane and nitriding," Journal of Materials Science, vol. 38, no. 4, pp. 689-692, 2003.

[16] C. J. Otten, O. R. Lourie, M. -F. Yu, et al., "Crystalline boron nanowires," Journal of the American Chemical Society, vol. 124, no. 17, pp. 4564-4565, 2002.

[17] Q. Yang, J. Sha, L. Wang, Z. Yuan, and D. Yang, "Aligned single crystal Al-catalyzed boron nanorods on Si substrates," European Physical Journal B, vol. 56, no. 1, pp. 35-39, 2007.

[18] T. T. Xu, J.-G. Zheng, N. Wu, et al., "Crystalline boron nanoribbons: synthesis and characterization," Nano Letters, vol. 4, no. 5, pp. 963-968, 2004.

[19] Z. Wang, Y. Shimizu, T. Sasaki, K. Kawaguchi, K. Kimura, and N. Koshizaki, "Catalyst-free fabrication of single crystalline boron nanobelts by laser ablation," Chemical Physics Letters, vol. 368, no. 5-6, pp. 663-667, 2003.

[20] D. Ciuparu, R. F. Klie, Y. Zhu, and L. Pfefferle, "Synthesis of pure boron single-wall nanotubes," Journal of Physical Chemistry B, vol. 108, no. 13, pp. 3967-3969, 2004.

[21] A. L. Pickering, C. Mitterbauer, N. D. Browning, S. M. Kauzlarich, and P. P. Power, "Room temperature synthesis of surface-functionalised boron nanoparticles," Chemical Communications, no. 6, pp. 580-582, 2007.

[22] Y.-S. Hu, Y. G. Guo, W. Sigle, S. Hore, P. Balaya, and J. Maier, "Electrochemical lithiation synthesis of nanoporous materials with superior catalytic and capacitive activity," Nature Materials, vol. 5, no. 9, pp. 713-717, 2006.

[23] T. Xu, C. Lin, C. Wang, D. L. Brewe, Y. Ito, and J. Lu, "Synthesis of supported platinum nanoparticles from Li-Pt solid solution," Journal of the American Chemical Society, vol. 132, no. 7, pp. 2151-2153, 2010.

[24] E. Weintraub, "On the properties and preparation of the element boron," Industrial and Engineering Chemistry, vol. 3, no. 5, pp. 299-301, 1911.

[25] C. L. Haertling, R. J. Hanrahan Jr., and J. R. Tesmer, "Hydrolysis studies of polycrystalline lithium hydride," Journal of Physical Chemistry C, vol. 111, no. 4, pp. 1716-1724, 2007.

[26] A. V. Vertkov, V. A. Evtikhin, and I. E. Lyublinski, "The vanadium alloys technological and corrosion studies in construction and operation of liquid metal facilities for fusion reactor," Journal of Nuclear Materials, vol. 233-237, no. 1, pp. 452-455, 1996.

[27] T. I. Serebryakova, V. I. Lyashenko, and V. D. Levandovskii, "Interaction in the system Li-B and some properties of lithium boride phases," Powder Metallurgy and Metal Ceramics, vol. 33, no. 1-2, pp. 49-53, 1995.

[28] P. Marmottant and S. Hilgenfeldt, "Controlled vesicle deformation and lysis by single oscillating bubbles," Nature, vol. 423, no. 6936, pp. 153-156, 2003.

[29] J. A. Rooney, "Other nonlinear acoustic phenomena," in Ultrasound Its Chemical, Physical, and Biological Effects, S. Suslick, Ed., pp. 74-96, VCH, New York, NY, USA, 1988.

[30] T. Scopigno, U. Balucani, A. Cunsolo, et al., "Phonon-like and single-particle dynamics in liquid lithium," Europhysics Letters, vol. 50, no. 2, pp. 189-195, 2000.

[31] D. Li, Y.-N. Xu, and W. Y. Ching, "Electronic structures, total energies, and optical properties of $\alpha$-rhombohedral $\mathrm{B}_{12}$ and tetragonal $\mathrm{B}_{50}$ crystals," Physical Review B, vol. 45, no. 11, pp. 5895-5905, 1992.

[32] S. Lee, D. M. Bylander, and L. Kleinman, "Bands and bonds of B $_{12}$," Physical Review B, vol. 42, no. 2, pp. 1316-1320, 1990. 

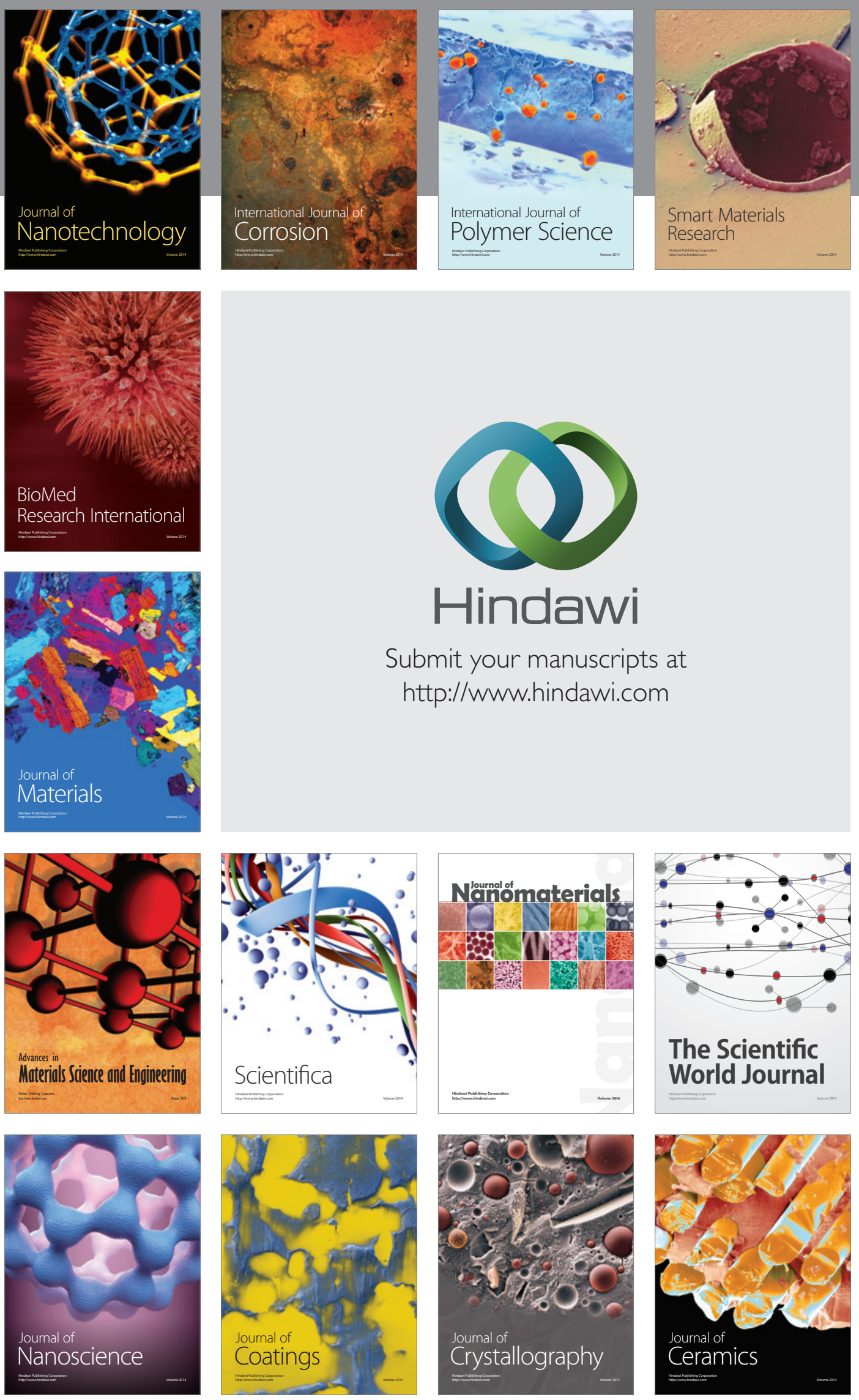

The Scientific World Journal

Submit your manuscripts at

http://www.hindawi.com

\section{World Journal}

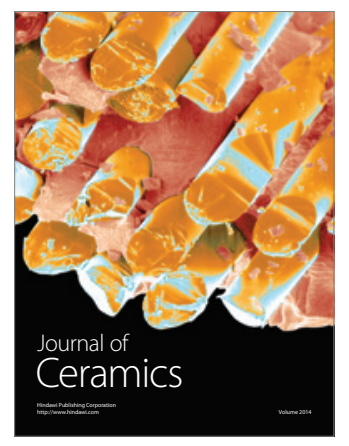

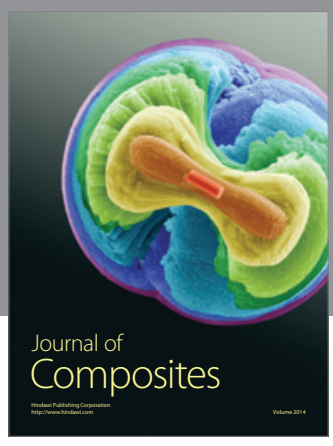
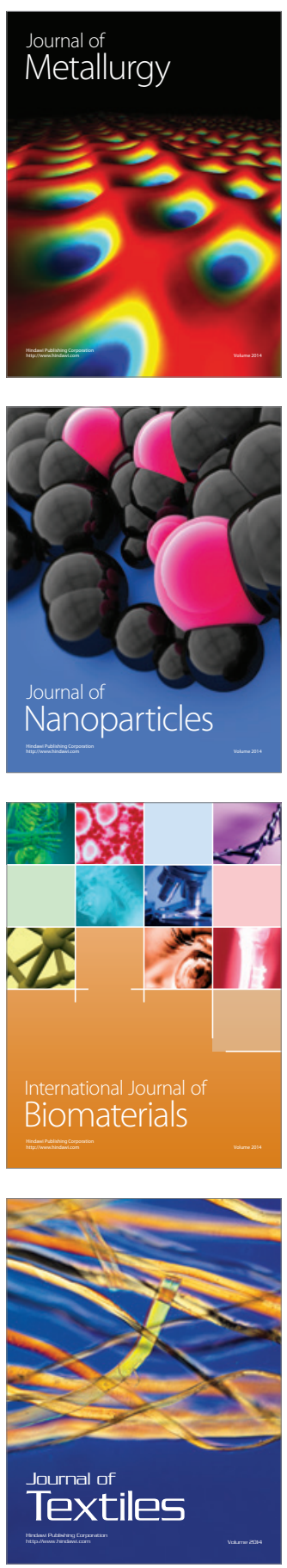